\title{
FACTS allocation considering loads uncertainty, steady state operation constraints, and dynamic operation constraints
}

\author{
M. M. H. Elroby ${ }^{1}$, S. F. Mekhamer ${ }^{2}$, H. E. A. Talaat ${ }^{3}$, M. A. Moustafa Hassan ${ }^{4}$ \\ ${ }^{1}$ Electrical Engineering Department, Faculty of Engineering, Ain Shams University, Egypt \\ ${ }^{2,3}$ Electrical Engineering Department, Future University, Egypt \\ ${ }^{4}$ Electrical Engineering Department, Cairo University, Egypt
}

\begin{tabular}{l} 
Article Info \\
\hline Article history: \\
Received Nov 14, 2019 \\
Revised Jul 11, 2020 \\
Accepted Jul 27, 2020 \\
\hline Keywords: \\
FACTS devices \\
Optimal power flow \\
Power system modeling \\
Steady state and dynamic \\
operation constraints \\
Uncertainty
\end{tabular}

\begin{abstract}
This study proposes an algorithm to allocate different types of flexible AC transmission system (FACTS) in power systems. The main objective of this study is to maximize profit by minimizing the system's operating cost including FACTS devices (FDs) installation cost. Dynamic and steady state operating restrictions with loads uncertainty are included in the problem formulation. The overall problem is solved using both teaching learning based optimization (TLBO) technique for attaining the optimal allocation of the FDs as main-optimization problem and matpower interior point solver (MIPS) for optimal power flow (OPF) as the sub-optimization problem. The validation of the proposed approach is verified by applying it to test system of 59-bus; Simplified 14-generator model of the South East Australian power system.
\end{abstract}

This is an open access article under the CC BY-SA license.

\section{Corresponding Author:}

M. M. H. Elroby

Electrical Engineering Department, Faculty of Engineering

Ain Shams University

Cairo, Egypt

Email: mousaelroby@yahoo.com

\section{INTRODUCTION}

Establishment of new transmission-lines (TLs) is arduous for the reasons of the environmental. Consequently, the TLs are loaded nearer to system security limits [1-3]. To ensure economic and secure operation, properly FACTS devices (FDs) allocation offers an effective means. During normal state, the objectives of the flexible AC transmission system (FACTS) can be relieving congestion, increasing voltage stability, increasing system loadability, and minimizing operating cost. During emergency states, the FACTS are used to fix the system. Each of the above stated objectives reinforce power system performance $[4,5]$. However, enhancement in one objective does not guarantee the same enhancement in others. Therefore, none of the stated technical objectives cannot be ignored in FDs allocation and should be formulated as multiobjective optimization problem.

Almost all current FACTS research works, try to improve power system steady-state characteristics. While power system dynamics should be considered in the multi-objective optimization problem. Furthermore, damping of the inter-area oscillations is considered as one of the significant appeals to the electric power system. The adjustment of power system stabilizer (PSS) settings does not sound to be the best way to treat inter-area oscillations, as the modes of these oscillation are not highly controllable and observable from measurements at the generating units [6]. Particularly, FACTS, flywheel, and battery storage play an important role in the inter-area damping oscillations [7]. These devices have the merit to be installed at any location in the power system, granting better performances compared to the PSS, which work 
proficiently for local oscillations comparatively to inter-area oscillations. To identify the best location of these devices, eigen value sensitivity and residue algorithm for the inter-area mode is analyzed [8]. The participation factors and residue method were used to determine FDs locations. Then, the controllers of the FACTS were designed based on their locations [9]. In [10], the concept of dynamic energy balance was used to model generator oscillations in different time-scales. In [11], an adaptive controller using model predictive control was proposed. With increasing of the phasor measurement units, the Controllers of the wide-area have been proposed to improve damping of the inter-area oscillations [6]. In [12] load-generation tripping was introduced as an active strategy for sustaining system from blackout.

Furthermore, various methods were proposed for power system optimization considering loads uncertainties. The perfect method is the Monte Carlo simulation (MCS). This method is usually used as standard method [13]. In [14], the MCS method for sizing of the multiple FDs in power systems to improve steady-state voltage profile was used. To reduce the cost of generation with taking into account uncertainty in load demand and renewable source, FACTS allocation was solved by MCS in coincidence with differential evolution algorithm [15].

The organization of this paper is as follows: Section 2 describes the slow coherency indices. Section 3 introduces the probabilistic optimal power flow (POPF). Section 4 introduce the model of the FACTS. Section 5 presents the optimal power flow. Section 6 presents the teaching learning based optimization (TLBO). Section 7 presents the statement of the problem. Section 8 discusses the applied case study. Finally, the conclusions are illustrated in section 9.

\section{THE SLOW COHERENCY INDICES}

The inter-area oscillations result when a coherent group of machines swings against each other groups. These oscillations are related with the weak TLs and larger loaded lines between the groups of tied coupled machines. These oscillations modes, if not properly damped, can lead to power system instability producing a complete blackout. The oscillation of the center angle of each area is slower than the oscillations between any two generators in the same area. This phenomenon occurs as a result of the strong tied between generators in the same area while between the areas the generators are weak tied [16]. Thus, the generators in the same areas interact on a "short-term basis", as they are coherent in the fast dynamic modes (fast coherent). Then, when the fast dynamics are decayed, the generators with different areas interact on a "longterm basis", as they are coherent in the slow dynamics (slow coherent). The coherency is used in the development of power system dynamic equivalents to simplify transient studies. The method for determining coherency of the power system is based on the simplified model with the following assumptions:

- The coherency can be evaluated based on linearized model, and the coherent areas are independent on amount of the disturbance.

- The coherent areas are independent of the generator models details. Therefore, the classical generator model can be considered and the turbine-governor and excitation can be ignored.

To identify coherency in power systems, the following equation is presented in [17].

$$
\Delta \ddot{\delta}=\mathrm{M}^{-1} \mathrm{~K} \Delta \delta
$$

where

$\mathrm{M}$ is the diagonal generator inertia matrix:

The $(i, j)$ element of $\mathrm{K}$ has the form

$$
K_{i j}=E_{i} E_{j}\left(B_{i j} \cos \left(\delta_{i}-\delta_{j}\right)-G_{i j} \sin \left(\delta_{i}-\delta_{j}\right)\right) \mathrm{|}_{\left(\delta_{0}, V_{0}\right)}, i \neq j
$$

$E_{i}$ is the voltage of generator $\mathrm{i}$,

$G_{i j}+j B_{i j}$ is the admittance between generator $i$ and $j$.

$K_{i i}=-\sum_{j=1, j \neq i}^{n} K_{i j}$, for the diagonal elements of $\mathrm{K}$

The entries $K_{i j}$ of $\mathrm{K}$ are the coefficients of the synchronizing-torque, as they keep the generator synchronized and stable. Thus, the stiffness of the connections between the areas are reduced, the slow dynamics are increased. The Eigen vectors of $M^{-1} K$ show shapes of the electromechanical modes. If generator $i$ and $j$ have similar values of the Eigen vector of $\lambda$ mode, we can deduce that these two generators are coherent with respect to that $\lambda$ mode. Therefore, if the eigen vectors matrix (Vs) correspond to the small eigen values of $M^{-1} K$, then a slow coherent group of generators have similar row in the eigen vectors matrix. Let the columns of eigen vectors matrix be normalized to unity, then the slow-coherency can be measured as follows [17]. 


$$
d_{i j}=w_{i} w_{j}^{T} /\left(\left|w_{i}\right|\left|w_{j}\right|\right)
$$

where $w_{i}$ and $w_{j}$ are the row of Vs corresponding to generators $i$ and $j$, respectively.

If generators $i$ and $j$ are perfectly coherent, then $w_{i}=w_{j}$ and $d_{i j}=1$. Therefore, the slow coherency indices $(\mathrm{SCI})$ that measures the slow coherency between the generators can be presented as follows:

$$
C I=\left\{\begin{array}{lcc}
C_{1} \sum_{j=2}^{n g}\left(1-d_{1 j}\right), & \text { for } & 0 \leq 1-d_{1 j} \leq 0.05 \\
+C_{2} \sum_{j=2}^{n g}\left(1-d_{1 j}\right), & \text { for } & 0.05<1-d_{1 j} \leq 0.1 \\
+C_{3} \sum_{j=2}^{n g}\left(1-d_{1 j}\right), & \text { for } & 0.1<1-d_{1 j} \leq 2
\end{array}\right.
$$

where

$\mathrm{ng}$ is the number of generators

$C_{1}, C_{2}, C_{3}$ are wighted constant and $C_{1}<C_{2}<C_{3}$

\section{PROBABILISTIC OPTIMAL POWER FLOW}

To encompass the majority of possible system states, it is necessary to run the deterministic power flow many times at different operating points. Hence, it turns out that it is appropriate to treat the deterministic power flow problem as a probabilistic power flow problem [18]. As quantities of the variables in the power system are treated as random variables (RVs), it becomes easy to determine the results ranges of the power flow. Several methods for POPF study have been done. These methods can be divided into three basic groups: approximate methods, MCS method, and analytical methods. MCS is a procedure that utilizes to solve a probabilistic problem. It is a strategy for iteratively estimating deterministic model. This strategy is regularly utilized when the model is nonlinear, complex, or has more parameters that are uncertain. The disadvantage of the MCS method is the huge number of the required samples to get convergence. However, the MCS technique is able to produce accurate results [19]. The point estimate method (PEM) is currently the representative of approximate methods for POPF calculations. The PEM was used to solve the POPF [18], [20]. The PEM like MCS use deterministic procedure to solve PPs. However, it requires a less computational encumbrance. Moreover, PEM overcome the awkwardness associated with the shortage of typical knowledge of the random variables, since these random variables are approximated by variance, mean, kurtosis, and skewness. Therefore, a least data is needed. The goal of any PEM is to determine the moments of the function that is a function of random variables. The used two-PEM (2PEM) in [21] is equivalent to Hong's $2 \mathrm{~m}$ scheme. The $2 \mathrm{PEM}$ does not give accurate results especially if the number of the input RVs is high. Therefore, it is not suitable for power system of actual size. However, the $2 \mathrm{~m}+1$ scheme is better than the $2 \mathrm{~m}$ scheme because it take into consideration the kurtosis of the input RVs while only one evaluation of the function is added [18]. Therefore, in this paper, the scheme $2 \mathrm{~m}+1$ is used to solve the POPF problem.

\section{1. $2 m+1$ Scheme}

This scheme requires $2 m+1$ evaluation of the function. Consequently, the weights and standard locations are [18]:

$$
\begin{aligned}
& p_{l . k}=\mu_{p l}+\zeta_{l, k} \sigma_{p l} \quad k=1,2,3 \\
& \zeta_{l, k}=\frac{\lambda_{l, 3}}{2}+(-1)^{3-k} \sqrt{\lambda_{l, 4}-\frac{3}{4} \lambda_{l, 3}^{2}} \quad k=1,2 \quad \zeta_{l^{\prime 3}}=0 \\
& w_{l . k}=\frac{(-1)^{3-k}}{m \zeta_{l, k}\left(\zeta_{l, 1}-\zeta_{l, 2}\right)} \quad k=1,2 \\
& w_{l .3}=\frac{1}{m}-\frac{1}{m\left(\lambda_{l, 4}-\lambda_{l, 3}^{2}\right)}
\end{aligned}
$$

where

$p_{l . k}$ is the locations of the input random variable $p_{l}$

$\zeta_{l, k}$ is the standard location

$\sigma_{p l}, \mu_{p 1}$, are the standard deviation and mean of the input random variable $p_{l}$,

$\lambda_{l, 4}$ and $\lambda_{l, 3}$ are the kurtosis and skewness of the input random variable $p_{l}$

FACTS allocation considering loads uncertainty, steady state operation constraints ... (M. M. H. Elroby) 
$w_{l . k}$ is weights of the locations $p_{l . k}$

From (4), setting $\zeta_{l^{3} 3}=0$ yields $p_{l .3}=\mu_{p l}$ and so, of the locations are the same $\left(\mu_{p 1}, \mu_{p 2}, \ldots, \mu_{p l}, \ldots, \mu_{p m}\right)$ point. Therefore, it is sufficient to run one evaluation of the probability function at this location, given that corresponding weight $w_{0}$ as follows:

$$
\mathrm{w}_{0}=1-\sum_{\mathrm{l}=1}^{\mathrm{m}} \frac{1}{\mathrm{~m}\left(\lambda_{\mathrm{l}, 4}-\lambda_{\mathrm{l}, 3}^{2}\right)}
$$

Furthermore, (4) shows that this scheme give non-real locations when $\lambda_{l, 4}-\frac{3}{4} \lambda_{l, 3}^{2}$ is negative value. However, in power system problems the probability distributions are usually utilized to binomial, uniform, or normal model, therefore the locations are permanently real values. To solve the POPF problem by $2 \mathrm{~m}+1$ scheme, the power flow input data are modeled as random variables, then the locations and weights are computed using (4) and (5). The solution of the POPF problem is presented in [18] where the idea can be explained using the (7):

$$
E\left(Z^{j}\right)=\sum_{l=1}^{m} \sum_{k=1}^{2} w_{l . k}(Z(l, k))^{j}+w_{0} Z_{0}^{j}
$$

where

$Z(l, k)$ is output of the probability function related to the kth concentration $\left(\mu_{p 1}, \mu_{p 2}, \ldots, p_{l, k}, \ldots, \mu_{p m}\right)$ of the input random variable $p_{l}$

$j$ is moments of the output

$Z_{0}$ is output of the probability function related to the kth concentration $\left(\mu_{p 1}, \mu_{p 2}, \ldots, \ldots, \mu_{p m}\right)$ of the input random variable $p_{l}$

The gross number of deterministic optimal power flow (OPF) to be run relies on the concentration schemes. The $Z(l, k)$ and $Z_{0}$ are used to evaluate the raw moments of the function output. The algorithm will ends when all concentrations of the all input RVs are considered. Then, the evaluated raw moments of the function output will be used to calculate the required statistical information using (7).

\section{4. $\quad$ FACTS model}

FDs technology includes a group of controllers that provide a possibility of controlling power system parameters, and it can be connected to a power system in various methods, such as in shunt, series, or a combination of shunt and series [22].

\subsection{Thyristor controlled series capacitor}

The TCSC can be considered as controlled reactance in series with the transsimission line. The main objective of this device is to compensate impedance of the TL. This compensation of the impedance can provide a control the power flow. This increases the system load ability and increases the damping of the interarea oscillation, and also provides a chance to quickly adjust power flow in response to the contingencies that may happen in the system [22]. The TCSC can be modelled as a variable reactance $\left(X_{T C S C}\right)$. TCSC implementation is executed through a fixed capacitor in parallel with a controlled reactor. Practically, it is recommended to compensate TL reactance up to a maximum compensating fraction (CF) of the TL reactance nominal value $\left(x_{k m}\right)$, both in inductive $\left(C F_{\text {ind }}\right)$ and the capacitive $\left(C F_{c a p}\right)$ operating zones. Therefore, The total branch reactance $\left(x_{T}\right)$ is as follows [22]:

$$
x_{T}=x_{k m}+x_{T C S C},
$$

$$
\text { Subject to: } C F_{\text {cap }} x_{k m}<x_{T}<C F_{\text {ind }} x_{k m}
$$

Practically, a rational $C F_{\text {cap }}$ and $C F_{\text {ind }}$ might be 0.7 and 1.3 of $x_{k m}$ respectively.

\subsection{Static synchronous compensator}

The static synchronous compensator (STATCOM) operates as a reactive power controlled source. The main feature of the STATCOM is to provide voltage support without using reactors and banks of capacitors in order to absorb or supply reactive power. Thus, the STATCOM has the ability to control its capacitive or inductive current independently of system voltage [22]. The STATCOM can inject or consume reactive power in the system bus. When the bus voltage is higher than the reference value, the STATCOM 
can consume reactive power from the network to decrease the bus voltage. In this case, the STATCOM is similar to an inductive behaviour. Further, when the bus voltage is less than the reference value, the STATCOM will injects reactive power into the bus sytem to increase the voltage, presenting a capacitive behaviour. The STATCOM can be represented by consumed or injected reactive power with working range of -300 to 300 MVAR.

\subsection{FACTS cost}

Once the optimum level for FDs is found, the cost of the TCSC and STATCOM can be calculated according to the following equations [23]:

$$
\begin{aligned}
& C_{S t a t}=0.0003 S^{2}-0.3051 S+127.38 \quad U S \$ / K V A R \\
& C_{T C S C}=0.0015 S^{2}-0.7130 S+153.75 \quad U S \$ / K V A R
\end{aligned}
$$

where $\mathrm{S}$ is the operating range of the FACTS devices in M VAR.

To compare the anticipated benefits against the cost of FDs, they have to be changed to the same unit. In this study, the comparison is made by changing the FDs cost into cost per Hour "US\$/h". To compute cost per Hour the following assumptions are made: Lifetime of the project $(\mathrm{n})=5$ years, Discount rate $(\mathrm{r})=10 \%$. The cost of FDs in US\$/h can be calculated as in [24]:

$$
F C_{h}=\frac{\left(C_{S t a}+C_{T C S C}\right) \times S \times 1000 \times \frac{r \times(1+r)^{n}}{(1+r)^{n}-1}}{8760}
$$

\section{OPTIMAL POWER FLOW}

In this study, the OPF is solved with the aid of matpower interior point solver (MIPS), which is coded in MATLAB 2017b [25]. MIPS are suited for very large-scale problems. In the success case, the solution in the feasible region, the solver generates flag equal to one. In the failure case, the solution in the infeasible region, the solver generates flag equal to zero. Therefore, if the flag equals to zero, the infeasibility case, the constraints can be considered as a normalized penalty functions as follows:

\subsection{Generators active power violation limits presented by}

$$
P g c=\sum_{i \in N g} P g c_{i}
$$

where

$$
P g c_{i}= \begin{cases}0 & \text { if } \underline{P g_{i}} \leq P g_{i} \leq \overline{P g_{i}} \\ \frac{P g_{i}}{P g_{i}}-1 & \text { if } P g_{i} \geq \overline{P g_{i}} \\ 1-\frac{P g_{i}}{\underline{P g_{i}}} & \text { if } P g_{i} \leq \underline{P g_{i}}\end{cases}
$$

$P g_{i}, \overline{P g_{i}}$ and $P g_{i}$ are generated, upper, and lower active power respectively for generator i.

\subsection{Generators reactive power violation limits presented by}

$$
Q g c=\sum_{i \in N g} Q g c_{i}
$$

where

$$
Q g c_{i}= \begin{cases}0 & \text { if } \underline{Q g_{i}} \leq Q g_{i} \leq \overline{Q g_{i}} \\ \frac{Q g_{i}}{\overline{Q g_{i}}}-1 & \text { if } Q g_{i} \geq \overline{Q g_{i}} \\ 1-\frac{Q g_{i}}{Q g_{i}} & \text { if } Q g_{i} \leq \underline{Q g_{i}}\end{cases}
$$

$\mathrm{Qg}_{\mathrm{i}}, \overline{\mathrm{Qg}_{\mathrm{i}}}$ and, $\mathrm{Qg}_{\mathrm{i}}$ are generated, upper, and lower reactive power respectively for generator $\mathrm{i}$. 


\subsection{Bus voltage violation limits presented by}

$$
V c=\sum_{i \in N b} V c_{i}
$$

where

$$
V c_{i}= \begin{cases}0 & \text { if } \underline{V_{i}} \leq V_{i} \leq \overline{V_{i}} \\ \frac{V_{i}}{\overline{V_{i}}}-1 & \text { if } V_{i} \geq \overline{V_{i}} \\ 1-\frac{V_{i}}{\underline{V_{i}}} & \text { if } V_{i} \leq \underline{V_{i}}\end{cases}
$$

$\mathrm{V}_{\mathrm{i}}, \mathrm{V}_{\mathrm{i}}, \overline{\mathrm{V}}_{\mathrm{i}}$ are bus voltage, bus lower voltage, and bus upper voltage respectively for bus i.

\subsection{Flow violation limits presented by}

$$
I c=\sum_{i \in N t} I c_{i}
$$

where

$$
I c_{i}= \begin{cases}0 & \text { if } \underline{I_{i}} \leq I_{i} \leq \overline{I_{i}} \\ \frac{I_{i}}{\overline{I_{i}}}-1 & \text { if } I_{i} \geq \overline{I_{i}}\end{cases}
$$

$\mathrm{I}_{\mathrm{i}}$, and $\overline{\mathrm{I}_{\mathrm{i}}}$ are the line current and upper line current respectively for line $\mathrm{i}$.

\section{TEACHING-LEARNING BASED OPTIMIZATION}

TLBO technique depends on the influence of the teacher on learners in class. Similar to other population-based techniques, it uses a population to get the global solution. The process of the TLBO is splitted into two phases. The first phase is "Teacher phase" and the second phase is "Learner phase". In the "Teacher phase", the Teacher is considered the most learned student in the class. Therefore, at each iteration ' $i$ ', the best learned among the population will act as Teacher and denoted by $M_{\text {new }}$ and tries to bring the mean of the class $(M i)$ towards his level. The solution is improved using the difference mean described as follows [26]:

$$
\text { Difference Mean }=r i *(\text { Mnew }-T F * M i)
$$

where $r i$ is the random number within range $[0,1]$

$\mathrm{TF}$ is either 1 or 2 .

The old solution will be updated using the difference mean as follows:

$$
\text { Snew }, i=\text { Sold, } i+\text { Difference Mean }
$$

If Snew, i is better than the Sold, i, then accept Snew, $i$ otherwise keep it as it is.

In the "Learner phase", the student improves his knowledge up to a certain level. In this phase, the student will come to know new information by self-studying. In this phase, student updating is given:

Randomly, select two students $S i$ and $S j$ where $i \neq j$

$$
\begin{array}{ll}
\text { Snew, } i=\text { Sold }, i+r i(S i-S j) & \text { if } f(S i)<f(S j) \\
\text { Snew, } i=S o l d, i+r i(S j-S i) & \text { if } f(S i) \geq f(S j)
\end{array}
$$

If Snew, $i$ gives better solution than Sold, $i$ then, accept it otherwise keep as it is. The algorithm will continue until the abort condition is met. 


\section{PROBLEM STATEMENT}

The allocation of the STATCOM and TCSC is expressed as a mixed continues-discrete problem. The flowchart of the planned algorithm is shown in Figure 1. The overall problem is formulated as two levels. In the first level, the TLBO searches of location and rating of the FDs then the result of the first level is passed into the second level. In the second level, the problem is divided into two branches. The first branch (the right branch), the TLBO searches of the loads between the upper and lower value that return max value of the OPF violation limits (i.e. the worst case of the system violation limits) according to the (19):

$$
\mathrm{E}_{\mathrm{cmax}}=\max \left\{\mathrm{W}_{1}\left(\mathrm{Pgc}+\mathrm{Q}_{\mathrm{gc}}+\mathrm{Ic}+\mathrm{Vc}\right)+\mathrm{W}_{2} \mathrm{SCI}\right\}
$$

The second branch (the left branch) uses 2PEM and OPF for the evaluation of the expected value (i.e. moment one (7)) of generation $\operatorname{cost}\left(O P F_{g c_{\text {expc }}}\right)$. Then the worst case of the constraints $\mathrm{E}_{\mathrm{cmax}}$ and $O P F_{g c_{\text {expc }}}$ are returned to the first level to be consider in the objective function as follows:

$$
\mathrm{F}_{\min }=\min \left\{\mathrm{W}_{3}\left(O P F_{g c_{\text {expc }}}+\mathrm{C}_{\mathrm{h}}\right)+\mathrm{W}_{4} \mathrm{E}_{\mathrm{cmax}}\right\}
$$

It can be concluded that the algorithm searches of the best locations and ratings of the FDs that minimize generation and FDs cost for all possible loads and minimize the worst-case of the system violation limits.

\section{CASE STUDY}

The effectiveness of the proposed algorithm is demonstrated on the IEEE 14-Generator test system. The data of this power systems are available in [27]. All FDs are removed from system then it considered as a base case. The statistical data for this system is identified beforehand. For simplicity, the values of the skewness, standard deviation, and kurtosis for each load bus are set on 15\%, 0.3041, and 2.5392, respectively. In addition, the mean, the lower, and upper for each load bus are considered 0.8, 0.6, and 1.1 of the heavy case respectively. The proposed algorithm is used to allocate four STATCOM and four TCSC devices in the test system. The algorithm described in section 7 was used to calculate the locations and rating of the FDs. The right branch in the second level of the Figure 1 was used to investigate the worst case of the system violation limits in the case of the absence of FDs. Then the results of these two cases (i.e. with and without FDs) are shown in Table 1. The left branch in the second level of the Figure 1 was used to investigate the expected generation cost $\left(O P F_{g c}\right.$ expc $)$ in the case of the absence of FDs. Then the results of these two cases (i.e. with and without FDs) are shown in Table 2. In addition, Table 3 shows the results of the FACTS location and rating. The Figure 2 shows the convergence curve of the proposed algorithm. Figure 3 to Figure 5 show buses voltage and lines flow for the worst case of the system violation limits in the case of the presence and absence of FDs.Based on the results of the proposed algorithm, the FDs shown in Table 3 are used in the Simulink model to perform time-domain simulation test as follows:

a. Contingency case: A three-phase fault is occurred at the end of line 409-411 near bus 411 at time $=1$. The fault was cleared after $0.1 \mathrm{~s}$. The Figure 6 and Figure 7 show the simulation results of all generators frequencies of the contingency case for without FDs and with FDs cases.

b. Remarks:

- As expected, the total expected cost $\left(\mathrm{G}_{\text {expc }}\right)$ and Constraints deviation are small in the case with FDs than case without FDs.

- It is obvious that the system operate without considering FDs fail to maintain the inter-area oscillation when it is subjected to this contingency. However, when FDs are considered, the inter-area oscillation can be successfully damped. This demonstrated that the proposed algorithm could successfully handle contingencies and reduce total cost.

Table 1. The worst case of the system constraints violation limits

\begin{tabular}{cccccc}
\hline & Pgc & $\mathrm{Q}_{\mathrm{gc}}$ & Vc & Ic & SCI \\
\hline Without FDs & 0 & 0 & 0.43 & 0.23 & 0.56 \\
With FDs & 0 & 0 & 0 & 0 & 0.1 \\
\hline
\end{tabular}

Table 2. The expected generation cost

\begin{tabular}{ccc}
\hline & Without FDs & With FDs \\
\hline Method & OPFgc expected & OPFgc expected + Ch \\
2PEM & 853385.6 & 802185.6 \\
\hline
\end{tabular}


Table 3. FACTSs locations and ratings

\begin{tabular}{ccccc}
\hline & TCSC & \multicolumn{2}{c}{ STATCOM } \\
From & To & $\begin{array}{c}\text { Compensating } \\
\text { Fraction }(\mathrm{Cf})\end{array}$ & Location & $\begin{array}{c}\text { Reactive Power } \\
\text { Rating }\end{array}$ \\
\hline 308 & 307 & 0.8 & 212 & 210 \\
102 & 309 & 0.7 & 412 & 188 \\
205 & 416 & 0.8 & 313 & 170 \\
408 & 405 & 0.8 & 507 & 190 \\
\hline
\end{tabular}

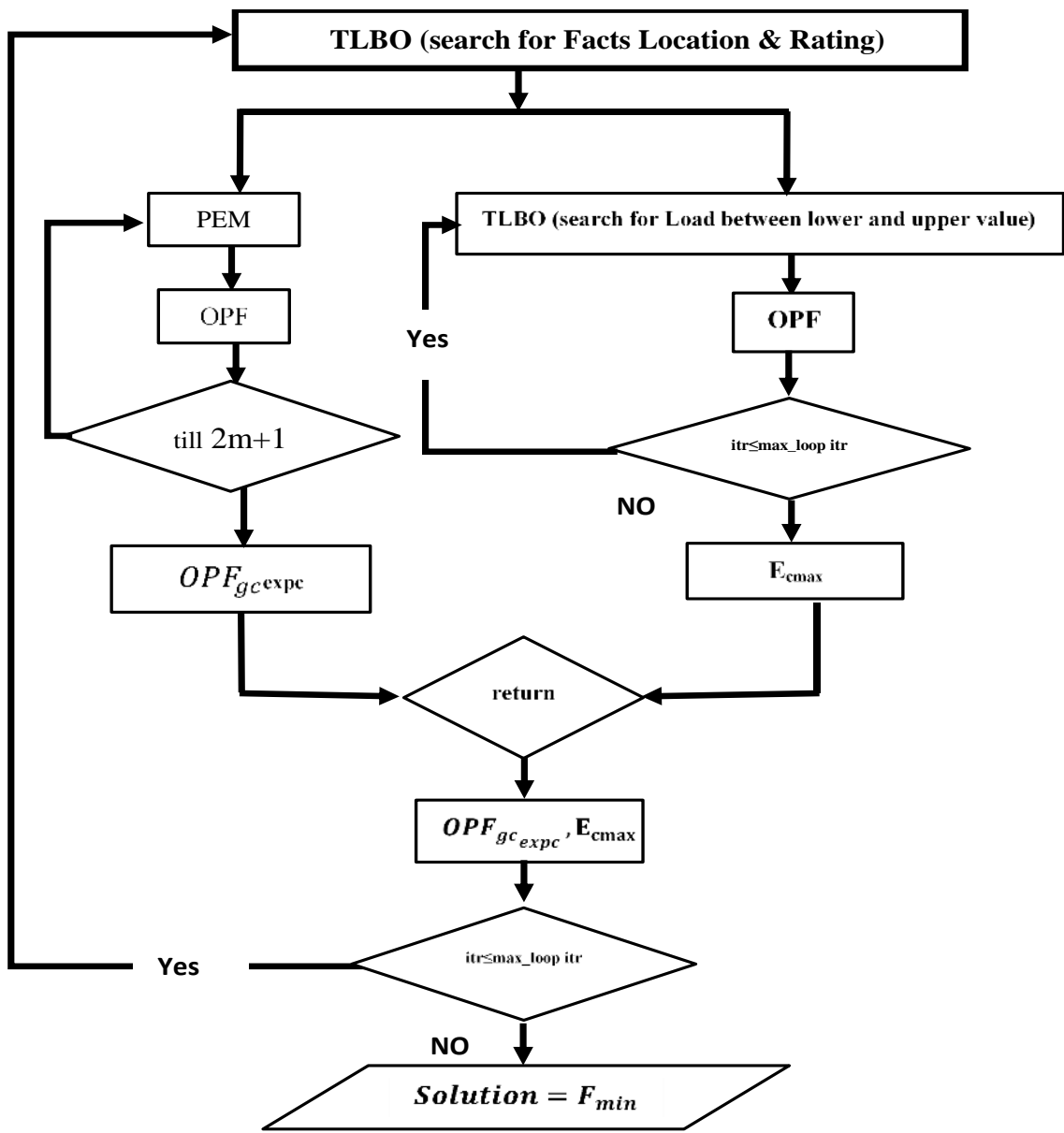

Figure 1. Flowchart of the FDs allocation

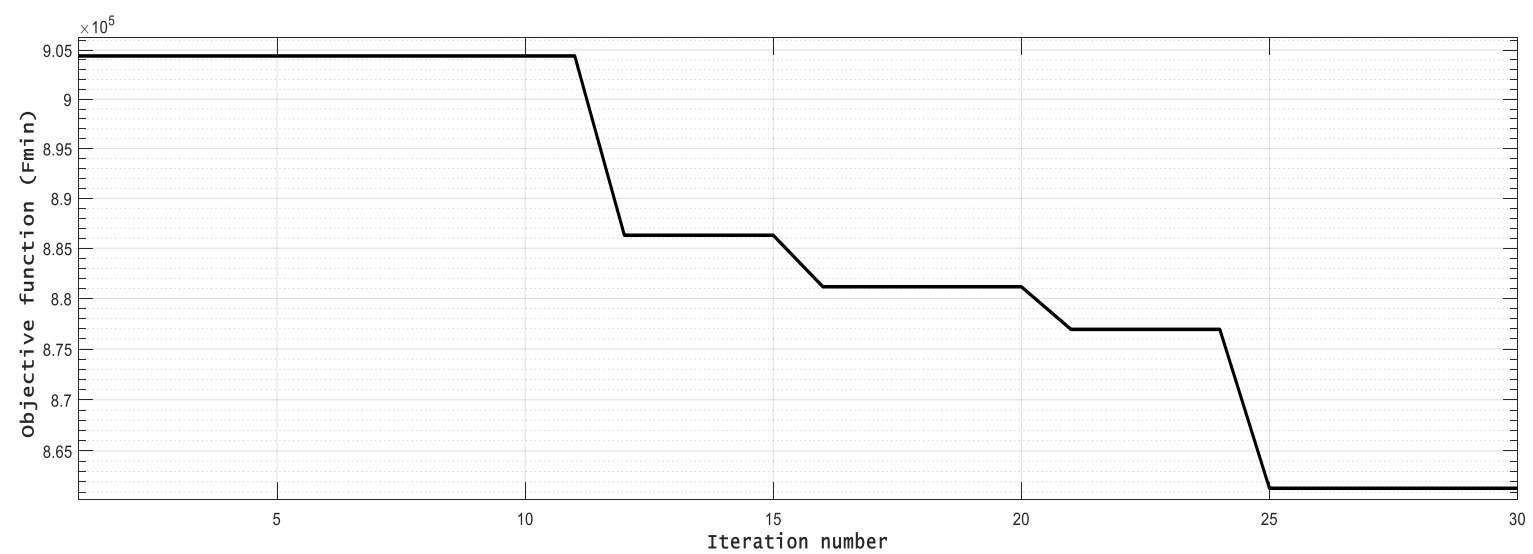

Figure 2. The convergence curve of the objective function for proposed algorithm 


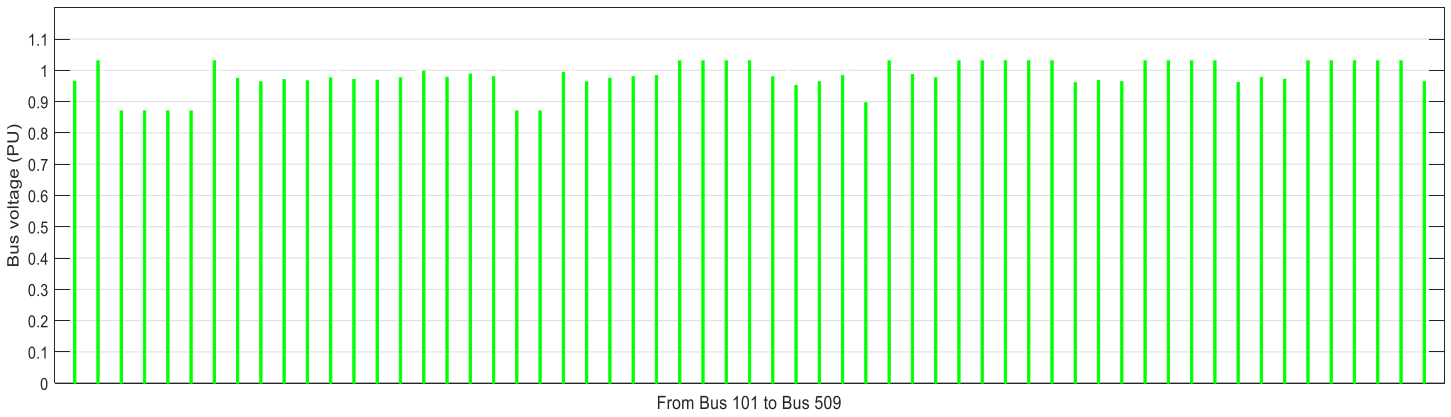

Figure 3. Test system buses voltage without FDs case

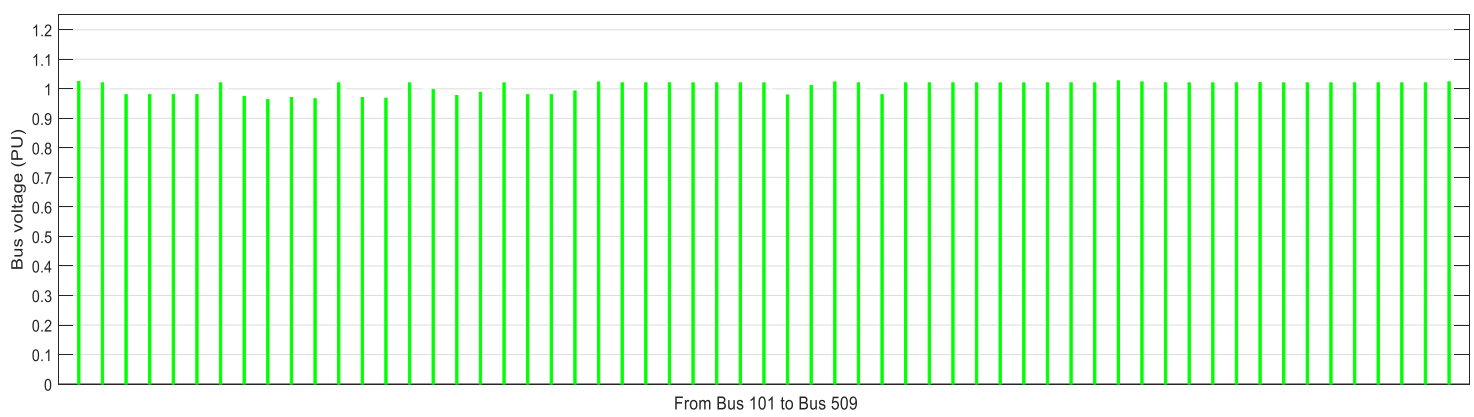

Figure 4. Test system buses voltage with FDs case

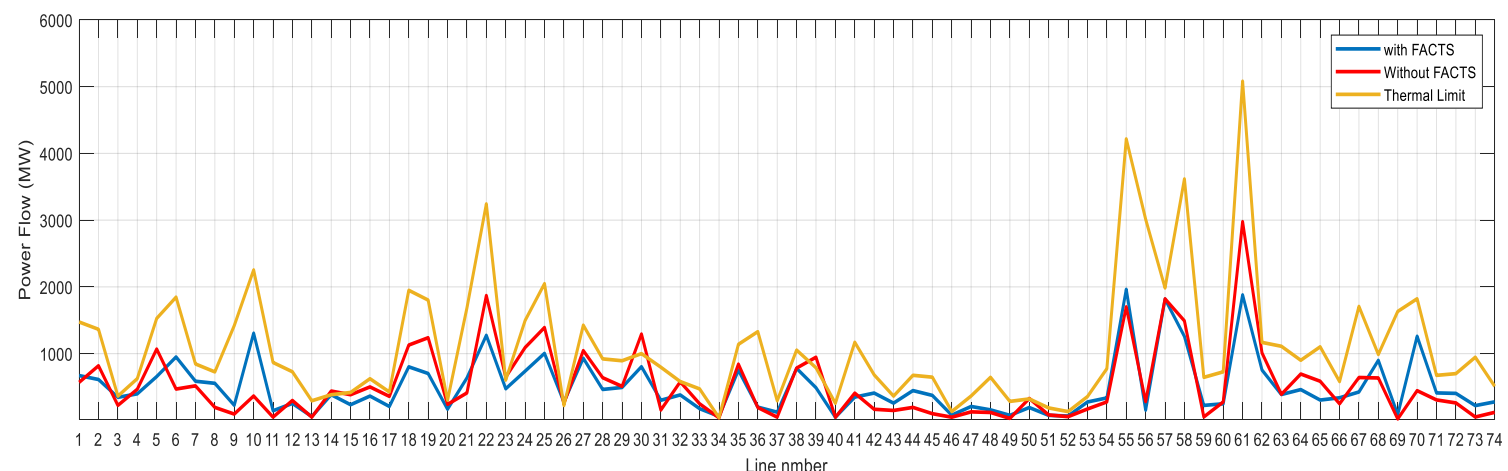

Figure 5. Lines flow with and without FDs case

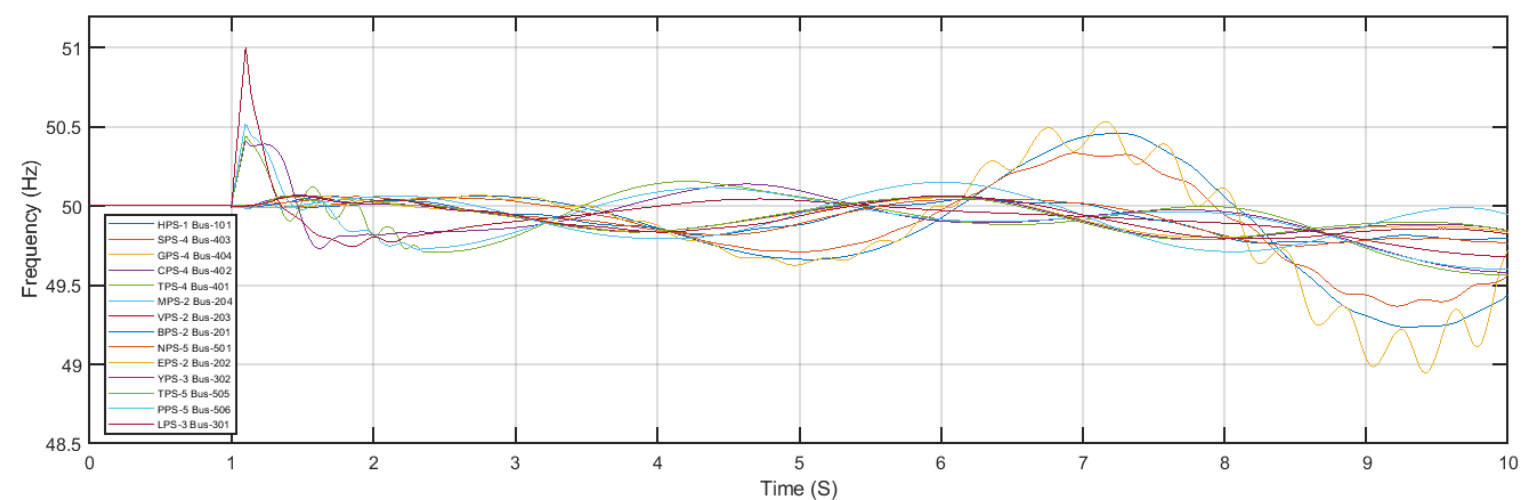

Figure 6. Simulation result of contingency fault without FDs 


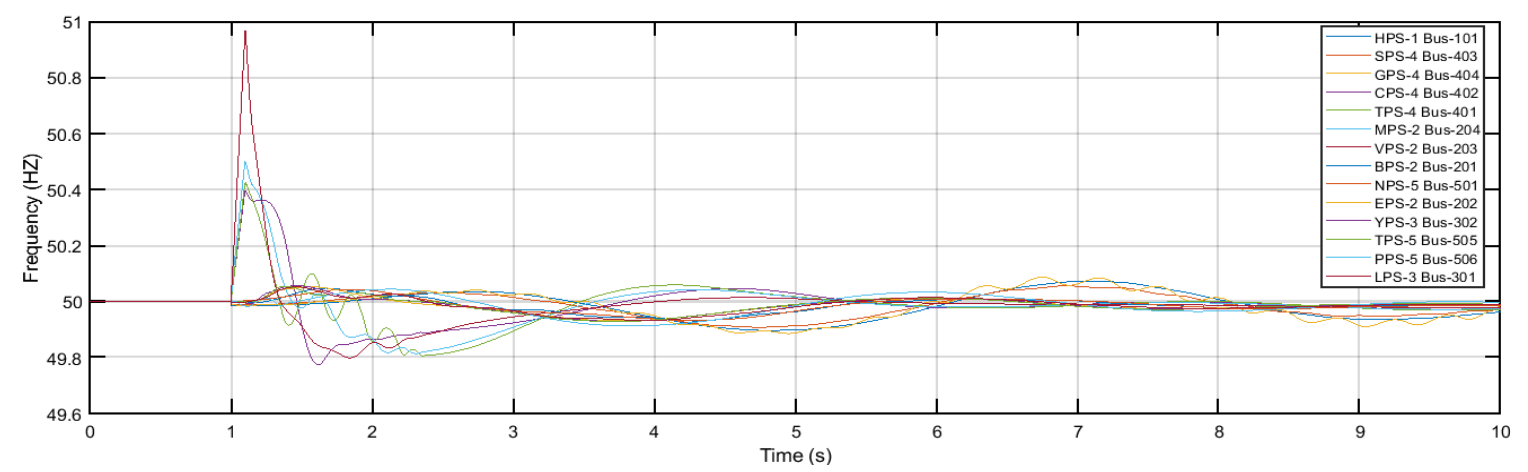

Figure 7. Simulation result of contingency fault with FDs

\section{CONCLUSION}

This study has presented an efficient and simple algorithm for determining the optimal location and capacity of the FDs. As evidenced by the case study, the proposed algorithm can successfully solve the challenging of contingency and is capable of providing better results in operation cost. The allocation of the FDs is presented as a multi-objective function. The objectives of the function are reducing the expected generation cost, steady-state constraints and improving the dynamic performance of the power system. The loads in this research are considered with the uncertainty model. The power system constraints are considered as counterpart problems (i.e. a minimum of maximum value) to achieve robust optimization. The effectiveness of the algorithm is investigated using the IEEE 59-bus test systems.

\section{REFERENCES}

[1] M. V. Rao, et al., "Available transfer capability evaluation and enhancement using various FACTS controllers: Special focus on system security," Ain Shams Engineering Journal, vol. 7, no. 1, pp. 191-207, 2016.

[2] S. Dawn, et al., "An approach for system risk assessment and mitigation by optimal operation of wind farm and FACTS devices in a centralized competitive power market," IEEE Transactions on Sustainable Energy, vol. 10, no. 3, pp. 1054-1065, 2019.

[3] A. Colmenar-Santos, et al., "Distributed generation: A review of factors that can contribute most to achieve a scenario of DG units embedded in the new distribution networks," Renewable and Sustainable Energy Review, vol. 59, pp. 1130-1148, 2016

[4] B. V. Kumar and N. V. Srikanth, "A hybrid approach for optimal location and capacity of UPFC to improve the dynamic stability of the power system," Applied Soft Computing, vol. 52, pp. 974-986, 2017.

[5] A. Elmitwally, et al., "Long-term economic model for allocation of FACTS devices in restructured power systems integrating wind generation," IET Generation, Transmission and Distribution, vol. 10, no. 1, pp. 19-30, 2016.

[6] W. Yao, et al., "Wide-Area Damping Controller for Power System Interarea Oscillations: A Networked Predictive Control Approach," IEEE Transactions on Control Systems Technology, vol. 23, no. 1, pp. 27-36, 2014.

[7] L. Yazdani and M. R. Aghamohammadi, "Damping inter-area oscillation by generation rescheduling based on wide-area measurement information," International Journal of Electrical Power and Energy Systems, vol. 67, pp. 138-151, 2015.

[8] M. Mandour, et al., "Damping of Power Systems Oscillations using FACTS Power Oscillation Damper-Design and Performance Analysis Damping of Power Systems Oscillations using FACTS Power Oscillation Damper-Design and Performance Analysis," 16th International Middle-East Power System Conference (MEPCON'2014) Ain Shams Univ. Cairo, Egypt, 2014, pp. 1-8.

[9] W. Yao, et al., "Wide-area damping controller of Facts devices for inter-area oscillations considering communication time delays," IEEE Transactions on Power System, vol. 29, no. 1, pp. 318-329, 2014.

[10] K. N. Stanton, "Dynamic energy balance studies for simulation of power-frequency transients," IEEE Transactions on Power Apparatus and Systems, vol. PAS-91, no. 1, pp. 110-117, 1972.

[11] H. Ye and Y. Liu, "Design of model predictive controllers for adaptive damping of inter-area oscillations," International Journal of Electrical Power and Energy System, vol. 45, no. 1, pp. 509-518, 2013.

[12] M. Khaji and M. R. Aghamohammadi, "Online emergency damping controller to suppress power system inter-area oscillation using load-generation tripping," Electric Power System Research, vol. 140, pp. 806-820, 2016.

[13] P. Jorgensen, et al., "Probabilistic load flow calculation using Monte Carlo techniques for distribution network with wind turbines," Proceedings of International Conference on Harmonicsand Quality of Power (ICHQP), vol. 2, 1998, pp. 1146-1151.

[14] S. O. Faried, et al., "Probabilistic technique for sizing FACTS devices for steady-state voltage profile enhancement," IET Generation, Transmission and Distribution, vol. 3, no. 4, pp. 385-392, 2009. 
[15] S. J. Galloway, et al., "Optimal flexible alternative current transmission system device allocation under system fluctuations due to demand and renewable generation," IET Generation, Transmission and Distribution, vol. 4, no. 6, pp. 725-735, 2010.

[16] W. J. H. Chow, et al., "Singular Perturbations, Coherency and Aggregation of Dynamic Systems," Final Report, U.So Dep. Energy Contrac, p. DE-ACO5-77ET2910.

[17] J. H. Chow, "Power System Coherency and Model Reduction," Power Electronics and Power Systems book series, Springer, vol. 94, 2013.

[18] J. M. Morales and J. Pérez-Ruiz, "Point estimate schemes to solve the probabilistic power flow," IEEE Transactions on Power Systems, vol. 22, no. 4, pp. 1594-1601, 2007.

[19] S. S. Reddy, et al., "Review of stochastic optimization methods for smart grid," Frontiers Energy, vol. 11, no. 2, pp. 197-209, 2017.

[20] M. El-Azab, et al., "A probabilistic multi-objective approach for FACTS devices allocation with different levels of wind penetration under uncertainties and load correlation," International Journal of Electrical Computer Engineering (IJECE), vol. 10, no. 4, pp. 3898-3910, 2020.

[21] S. Chun-Lien, "Probabilistic Load-Flow Computation Using Point Estimate Method," IEEE Transactions on Power Systems, vol. 20, no. 4, pp. 1843-1851, 2005.

[22] X. P. Zhang, et al., "Flexible AC transmission systems: Modelling \& control," Springer, 2006.

[23] D. Sen, et al., "Optimal allocation of static VAR compensator by a hybrid algorithm," Energy Systems, vol. 10, pp. 677-719, 2019.

[24] H. Hashemzadeh and S. H. Hosseini, "Locating series FACTS devices using line outage sensitivity factors and particle swarm optimization for congestion management," 2009 IEEE Power and Energy Society General Meeting (PES '09), 2009, pp. 1-6.

[25] R. D. Zimmerman and C. E. Murillo-s, "MATPOWER User's Manual Version 7.0b1," Power Systems Engineeering Research Center, 2019.

[26] D. P. Rao, et al., "Teaching-learning-based optimization: A novel method for constrained mechanical design optimization problems," Computer-Aided Design, vol. 43, no. 3, pp. 303-315, 2011.

[27] M. Gibbard and D. Vowles, "IEEE PES Task Force on Benchmark Systems for Stability Controls Simplified 14-Generator Model of the South East Australian Power System," The University of Adelaide, South Australia, 2014. 